\title{
LIMITATIONS AND CAPABILITIES OF FEEDBACK FOR CONTROLLING UNCERTAIN SYSTEMS*
}

\author{
L.-L. Xie and L. Guo \\ Institute of Systems Science \\ Chinese Academy of Sciences \\ Beijing, 100080, P.R.China \\ Lguo@iss03.iss.ac.cn
}

\begin{abstract}
Feedback is used primarily for reducing the effects of uncertainties on the performance of control systems, and the understanding of its limitations and capabilities is fundamental. This paper will present some preliminary results in this direction, showing that for a large class of discrete-time uncertain nonlinear stochastic systems, the growth rate of nonlinearities (GRN) is critical: if the GRN is faster than linear, then feedback stabilization is impossible in general even for systems with linear uncertain parameters; if, however, the GRN is linear, then an asymptotically optimal feedback can be constructed for a class of nonparametric uncertain systems.
\end{abstract}

\section{INTRODUCTION}

The main objective of using feedback in a control system design is to reduce the effects of the system uncertainties on the control performance. The uncertainties of a system usually stem from two sources: structure uncertainties and external disturbances (noises). In general, the later is easier to cope with than the former. A fundamental question in the area of systems and control is: What is the limitations and capabilities of feedback for controlling uncertain systems? This is a conundrum which only a few control areas could shed some light on. Robust control and adaptive control are two such areas where structure uncertainties of a system are the main concern in the controller design. Robust control usually requires that the true system lies in a small ball centered by a known nominal model (cf.[12]), whereas adaptive control does not need such a prerequest (cf.[1] [7]). Although much progress has been made in these

*Please address all correspondence to Professor Lei Guo. This work was supported by the National Natural Science Foundation of China and the National Key Project of China.

The original version of this chapter was revised: The copyright line was incorrect. This has been corrected. The Erratum to this chapter is available at DOI: 10.1007/978-0-387-35359-3_40 
two areas over the past two decades, the understanding of the fundamental question concerning about capabilities and limitations of feedback is far from being complete.

For linear finite dimensional systems with uncertain parameters, a welldeveloped theory of adaptive control exists today, both for stochastic systems (cf.[1] [4] [6]) and for deterministic systems with small unmodelled dynamics (cf.[7]). This theory can easily be generalized to nonlinear systems with linear unknown parameters and with linearly growing nonlinearities (cf.[9]). However, the generalization of the existing theory to systems with nonlinearities having nonlinear growth rates is possible only for continuous-time system (cf.[8]), not for discrete-time systems (cf.[5]). This is a fundamental difference between adaptive feedback control of discrete-time and continuous-time systems.

All the above mentioned results are concerned with parametric models with linear uncertain parameters. This is of course only a special situation. The more challenging problem is to control uncertain nonparametric systems. We now give it a little more detailed account. Let $f(\cdot)$ be an unknown nonparametric function describing the nonlinear dynamics of a control system. Various approximation techniques exist in the literature (e.g. Volterra series, fuzzy and neural nets, wavelets, etc.), which basically state that for $x$ in a compact set, $f(\cdot)$ can be uniformly approximated by parametric functions of the form

$$
g(\theta, x) \triangleq \sum_{i=1}^{N} a_{i} \sigma\left(b_{i}^{\tau} x\right),
$$

where $\sigma(\cdot)$ is a known "basis" function, $a_{i}$ 's and $b_{i}$ 's are unknown parameters or weights denoted by $\theta$, and $N$ is an integer reflecting the complexity/accuracy of approximation.

Thus, one may conceive that the above explicit parametric model $g(\theta, x)$ can be used in adaptive control instead of using the original nonparametric model $f(\cdot)$. This natural idea has indeed attracts considerable attention from researches (e.g. [11]). This approach, though attractive and effective in some applications, has several fundamental limitations/difficulties. First, in order to ensure that $x$ (which usually represents the system state or output signals) lies in a compact set for reliable approximation, stability of the system must be established first, and the parametric model provides little (if any) help in this regard; Second, searching for the optimal parameters $a_{i}$ 's and $b_{i}$ 's usually involves in global nonlinear optimization, of which a general efficient scheme is still lacking by now, and moreover, the on-line combination of the estimation and control (adaptive control) will further complicate the problem; Third, no matter how large the approximation complexity $N$ is, there always exists an approximation error in the model, which will inevitably prevent the parametricmodel-based control to be optimal in general. Hence, it is of advantages to consider the nonparametric model $f(\cdot)$ directly, and it is natural to use the nonparametric estimation methods which are well-developed in the mathematical statistics literature (cf. [2] and the references therein). To the best of the authors' knowledge, the first concrete theoretical result on nonparametric 
adaptive control seems to have been obtained by Oulidi (see [2]), who proved that the diminishingly excited certainty equivalence nonparametric adaptive control is asymptotically optimal for systems with bounded noises.

The understanding of the limitations and capabilities of feedback for controlling uncertain systems is inextricably linked to the puzzling question: how much of the uncertainties can be predicted and reduced based on the available information? Clearly, it is more convenient to answer this "predictability" question in the stochastic framework, since the concept of "conditional expectation" in probability theory is a natural, suitable and powerful tool.

In this paper, we shall present some preliminary yet concrete results on the limitations and capabilities of feedback in the presence of structure uncertainties of the systems to be controlled. We shall first show that even in the case of linear uncertain parameters, feedback control may still not be able to stabilize the system if the nonlinearities have a nonlinear growth rate in general. Then we will show that if the linear growth rate constraint is imposed, then a feedback controller can be constructed to control a large class of nonparametric uncertain systems in an asymptotically optimal way, without resorting to any external excitations.

\section{LIMITATIONS OF FEEDBACK FOR STABILIZING UNCERTAIN NONLINEAR SYSTEMS}

Consider the following typical discrete-time polynomial nonlinear regression model

$$
y_{t+1}=\theta_{1} y_{t}^{b_{1}}+\theta_{2} y_{t}^{b_{2}}+\cdots+\theta_{n} y_{t}^{b_{n}}+u_{t}+w_{t+1}, \quad t \geq 0
$$

where $y_{t}$ and $u_{t}$ are the system output and input signals respectively, $\theta_{i}(1 \leq$ $i \leq n$ ) are unknown parameters and $w_{t}$ is the noise signal.

Assume that

(A1) $b_{i}(1 \leq i \leq n)$ are nonnegative real numbers making (1.1) meaningful and satisfying

$$
b_{1}>b_{2}>\cdots>b_{n}>0
$$

(A2) $\left\{w_{t}\right\}$ is a Gaussian white noise sequence with distribution $N(0,1)$;

(A3) The unknown parameter vector $\theta \triangleq\left[\theta_{1}, \cdots, \theta_{n}\right]^{\tau}$ is independent of $\left\{w_{t}\right\}$ and has a Gaussian distribution $N\left(\bar{\theta}, I_{n}\right)$.

Our objective is to study the global stabilizability of (1.1) under the above conditions. First, we give a precise definition of stabilizability.

Definition 2.1 Let $\sigma\left\{y_{i}, 0 \leq i \leq t\right\}$ be the $\sigma$-field generated by the observations $y_{i}, 0 \leq i \leq t$. The system $(1.1)$ is said to be a.s. globally stabilizable, if there exists a feedback control

$$
u_{t} \in \mathcal{F}_{t}^{y} \triangleq \sigma\left\{y_{i}, 0 \leq i \leq t\right\}, \quad t=0,1, \cdots
$$

such that for any initial condition $y_{0} \in R^{1}, \limsup _{T \rightarrow \infty} \frac{1}{T} \sum_{t=1}^{T} y_{t}^{2}<\infty$, a.s. 
Remark 2.1 We remark that the global stabilization of (1.1) is a trivial task in either the case where $\theta$ is known or the case where the noise is free (i.e. $w_{t} \equiv 0$ ). To be precise, if $\theta$ were known, we can put $u_{t} \equiv-\left(\theta_{1} y_{t}^{b_{1}}+\theta_{2} y_{t}^{b_{2}}+\cdots+\theta_{n} y_{t}^{b_{n}}\right)$, which gives $y_{t+1} \equiv w_{t+1}$, and the system is stabilized since

$$
\limsup _{T \rightarrow \infty} \frac{1}{T} \sum_{t=1}^{T} y_{t}^{2}=\limsup _{T \rightarrow \infty} \frac{1}{T} \sum_{t=1}^{T} w_{t}^{2}<\infty .
$$

In the case where $\theta$ is unknown but the noise is free $\left(w_{t} \equiv 0\right)$, we can obtain the true value of the parameter $\theta$ by solving $n$ linear independent equations. For example, if in the first $(n+1)$ steps, we choose $\left\{u_{t}, 0 \leq t \leq n\right\}$ to be independently identically distributed random variables with probability density function $p(x)$, then it is not difficult to prove the nonsingularity of the following matrix:

$$
A \triangleq\left[\begin{array}{llll}
y_{1}^{b_{1}} & y_{1}^{b_{2}} & \ldots & y_{1}^{b_{n}} \\
y_{2}^{b_{1}} & y_{2}^{b_{2}} & \ldots & y_{2}^{b_{n}} \\
\vdots & \vdots & \ddots & \vdots \\
y_{n}^{b_{1}} & y_{n}^{b_{2}} & \cdots & y_{n}^{b_{n}}
\end{array}\right]
$$

Hence the true value of the parameter $\theta$ can easily be obtained by solving the following linear equation derived by rearranging (1.1):

$$
A \cdot \theta=\left[y_{2}-u_{1}, y_{3}-u_{2}, \cdots, y_{n+1}-u_{n}\right]^{\tau} \text {. }
$$

Then again we can take the control as $u_{t}=-\left(\theta_{1} y_{t}^{b_{1}}+\theta_{2} y_{t}^{b_{2}}+\cdots+\theta_{n} y_{t}^{b_{n}}\right)$ for $t>n$, which globally stabilizes the noise-free system. For more general parametric-strict-feedback models with no noises, a similar approach can also be applied to design a globally stabilizing adaptive controller regardless of the growth rate of the nonlinearities.

Unfortunately, the main drawback of the above approach is that the resulting adaptive controller is not robust with respect to noises. In fact, the presence of noises will change the stabilizability of discrete-time nonlinear systems dramatically if the growth rate of the nonlinearities is faster than linear, as will be shown by the following theorem together with its corollaries.

Theorem 2.1 ([10]) Under Assumptions (A1)-(A3), the system (1.1) is not a.s. globally stabilizable by feedback whenever the following inequality

$$
P(z)<0, \quad z \in\left(1, b_{1}\right)
$$

has a solution, where $P(z)$ is a polynomial defined by

$$
P(z)=z^{n+1}-b_{1} z^{n}+\left(b_{1}-b_{2}\right) z^{n-1}+\cdots+\left(b_{n-1}-b_{n}\right) z+b_{n} .
$$

To understand the implications of Theorem 2.1, we now give some detailed discussions on the inequality (1.5). 
Corollary 2.1 ([10]) Let $b_{i}(1 \leq i \leq n)$ satisfy

$$
b_{1}>1 \text { and } 0<b_{i}-b_{i+1} \leq \frac{\sqrt{b_{1}}}{2}\left(\sqrt{b_{1}}-1\right)^{2}, \quad 1 \leq i \leq n-1
$$

then (1.5) has a solution whenever $n \geq 2 \log \left(\frac{\sqrt{b_{1}}+1}{\sqrt{b_{1}}-1}\right) / \log b_{1}$. Consequently, whenever $b_{1}>1$ and the number of unknown parameters $n$ is suitably large, there always exist $0<b_{n}<b_{n-1}<\cdots<b_{1}$ such that (1.1) is not a.s. globally stabilizable.

Remark 2.2 By Corollary 2.1 we know that the usual linear growth condition imposed on the nonlinear function $f(\cdot)$ of the general control model

$$
y_{t+1}=\theta^{\tau} f\left(y_{t}\right)+u_{t}+w_{t+1}, \quad \theta \in R^{n}
$$

cannot be essentially relaxed in general for global adaptive stabilization, unless additional conditions on the number $n$ and the structure of $f(\cdot)$ are imposed. Remark 2.3 Let us consider the following continuous-time counter-part model

$$
d y_{t}=\left[\theta^{\tau} f\left(y_{t}\right)+u_{t}\right] d t+d w_{t}, \quad t \geq 0,
$$

where $\theta \in R^{p}$ is an unknown parameter vector, and $f(x): R^{1} \rightarrow R^{p}$ is a continuous function satisfying the local Lipschitz condition, and $\left\{w_{t}\right\}$ is a standard Brownian motion. Assume that $\|f(x)\| \leq L_{1}+L_{2}|x|^{k}$ for some integer $k>0$ and for some constants $L_{1}, L_{2}>0$. Then it can be shown that the following feedback control of nonlinear damping type:

$$
u_{t}=-c y_{t}-y_{t}^{2 k+1}, \quad c>0
$$

can stabilize the systems regardless of the growth rate of the nonlinearities (measured by $k$ ).

The above two remarks demonstrate the fundamental difficulties between feedback stabilizability of discrete- and continuous-time uncertain systems.

Corollary 2.2 Let $b_{1}>2$, then for $n>1+2 \log \left(\frac{2}{b_{1}-2}\right) / \log \left(\frac{b_{1}}{2}\right)$, has a solution for any $\left\{b_{i}\right\}$ satisfying $1 \leq b_{n}<b_{n-1}<\cdots<b_{2}<b_{1}$. On the other hand, if $b_{1} \leq 2$, then for any $n$, there always exist $1 \leq b_{n}<b_{n-1}<\cdots<$ $b_{2}<b_{1}$ such that (1.5) has no solution.

Corollary 2.3 For any $n \geq 1$ and any $b_{1}>b_{2}>\cdots>b_{n}>0$,

(i) A necessary condition for (1.5) to have a solution is $\sum_{i=1}^{n} b_{i}>4$;

(ii) A sufficient condition for (1.5) to have a solution is either $b_{1}>4$, or

$$
\sum_{i=1}^{n} b_{i}>(n+1)\left(1+\frac{1}{n}\right)^{n}
$$

The above three corollaries give us a picture concerning about situations where the nonlinear model (1.1) is not a.s. globally stabilizable by feedback. 


\section{OPTIMAL FEEDBACK CONTROL OF NONPARAMETRIC UNCERTAIN SYSTEMS}

In this section, we are going to show that if the nonlinearities have a certain linear growth rate, then optimal feedback control can be designed even for the following uncertain nonparametric model:

$$
y_{t+1}=f\left(y_{t}\right)+u_{t}+\varepsilon_{t+1},
$$

where $y_{t}, u_{t}$ and $\varepsilon_{t}$ are the $d$-dimensional system output signals, input signals and white noises, and $f(\cdot)$ is an unknown nonlinear function.

Our objective is to design a feedback control $u_{t}$ based on the observations $\left\{y_{i}, i \leq t\right\}$ at each step $t$, such that the system output $\left\{y_{t}\right\}$ tracks a known reference signal $\left\{y_{t}^{*}\right\}$ in an optimal way. If $f(\cdot)$ were known, it is obvious that such a controller would take the following form:

$$
u_{t}=-f\left(y_{t}\right)+y_{t+1}^{*} \text {. }
$$

Since in the present case, $f(\cdot)$ is unknown, we adopt the nonparametric estimation approach as used in [2], but without resorting to the external excitations used there.

Let $K(\cdot)$ be a nonnegative kernel function satisfying the following conditions:

$$
K(0)>0, \quad \int K(s) d s=1, \quad \int K^{2}(s) d s<\infty, \quad \int\|s\| K(s) d s<\infty .
$$

Here in our estimation process, let $K(\cdot)$ have a compact support, i.e.,

$$
K(s)=0, \quad \text { for }\|s\|>A .
$$

Let $\delta(\cdot, \cdot)$ be a function shifted from $K(\cdot)$ :

$$
\delta_{j}(x, y) \triangleq K\left(j^{a}(x-y)\right), \quad \forall j>0, \quad \delta_{0}=0,
$$

where $a \in\left(0, \frac{1}{2 d}\right), d$ is the dimension of the system signals.

The nonparametric estimate of $f(y), y \in \mathbb{R}^{d}$ at time $\mathrm{t}$ is defined by

$$
\widehat{f}_{t}(y)= \begin{cases}N_{t}^{-1}(y) \sum_{j=1}^{t} \delta_{j-1}\left(y_{j-1}, y\right)\left(y_{j}-u_{j-1}\right), & \text { if } N_{t}(y)>0 \\ 0 & \text { otherwise }\end{cases}
$$

where,

$$
N_{t}(y) \triangleq \sum_{j=1}^{t} \delta_{j-1}\left(y_{j-1}, y\right)
$$


To define the adaptive feedback control, we need to introduce a sequence of truncation bounds denoted by $\left\{h_{t}\right\}$, which is positive, monotonically diverges to infinity, and satisfies

$$
h_{t}=o(\sqrt{\log t}), \text { as } t \rightarrow \infty .
$$

Now, by the (truncated) certainty equivalence principle, the nonparametric adaptive control can be defined as

$$
u_{t}=-\widehat{f}_{t}\left(y_{t}\right) I_{\left(\left|\widehat{f}_{t}\left(y_{t}\right)\right| \leq h_{t}\right)}+y_{t+1}^{*}
$$

where $I_{(\cdot)}$ is the indicator function.

With this control, the closed-loop system equation is

$$
y_{t+1}=f\left(y_{t}\right)-\widehat{f}_{t}\left(y_{t}\right) I_{\left(\left|\widehat{f}_{t}\left(y_{t}\right)\right| \leq h_{t}\right)}+y_{t+1}^{*}+\varepsilon_{t+1},
$$

which is obviously a nonlinear dynamical system.

In order to analyze the properties of (1.15), we introduce the following assumptions on the system (1.9):

(A4) The nonlinear function $f(\cdot)$ is Lipschitz continuous, and there exist two constants $\alpha \in(0,1)$ and $\beta \in(0, \infty)$ such that

$$
\|f(x)\| \leq \alpha\|x\|+\beta, \quad \forall x \in \mathbb{R}^{d} .
$$

(A5) $\left\{\varepsilon_{t}\right\}$ is a Gaussian white noise sequence with mean zero and variance $\sigma^{2}>0$.

(A6) The reference signal $\left\{y_{t}^{*}\right\}$ is bounded.

Theorem 3.1 Consider the control system (1.9) where the nonlinear function $f(\cdot)$ is completely unknown. Let the assumptions (A4)-(A6) be fulfilled. Then the adaptive feedback tracking control defined by (1.14) is asymptotically optimal in the sense that

$$
\lim _{T \rightarrow \infty} \frac{1}{T} \sum_{t=1}^{T}\left(y_{t}-y_{t}^{*}\right)^{2}=\sigma^{2}, \quad \text { a.s. }
$$

The proof may be found in [3].

\section{CONCLUDING REMARKS}

In this paper, we have presented several preliminary results concerning limitations and capabilities of feedback for controlling uncertain nonlinear systems. Of course, this is just a standing point towards a more comprehensive theory. Many interesting problems still remain open, among which we only mention the following two: (i) If in Theorem 2.1 the inequality (1.5) has no solution, can we construct a stabilizing feedback? (ii) Is it possible to remove the stringent condition $\alpha \in(0,1)$ in Condition (A4) of Theorem 3.1? 


\section{References}

[1] H. F. Chen and L. Guo, Identification and Stochastic Adaptive Control, Boston, MA: Birkhäuser, 1991.

[2] M. Duflo, Random Iterative Models, Berlin: Springer-Verlag, 1997.

[3] L. Guo and L. L. Xie, An optimal nonparametric adaptive control without external excitation, Preprints of the 14th IFAC World Congress, Beijing, July 5-9, 1999. (To appear).

[4] L. Guo, Self-convergence of weighted least-squares with applications to stochastic adaptive control, IEEE Trans. Automat. Contr., Vol.41, No.1, pp.79-89, 1996.

[5] L. Guo, On critical stability of discrete-time adaptive nonlinear control, IEEE Trans. Automat. Contr., Vol.42, No.11, pp.1488-1499, 1997.

[6] L. Guo and H. F. Chen, The Astrom-Wittenmark self-tuning regulator revisited and ELS-based adaptive trackers, IEEE Trans. Automat. Contr., Vol.36, No.7, pp.802-812, 1991.

[7] P. A. Ioannou and J. Sun, Robust Adaptive Control, NJ: Prentice-Hall, 1996.

[8] M. Krstic, I. Kanellakopoulos and P. V. Kokotovic, Nonlinear and Adaptive Control Design, New York: John Wiley \& Sons, 1995.

[9] C. Wei and L. Guo, Adaptive control of a class of nonlinear stochastic systems, Proc. 1996 Chinese Control Conference, pp.508-512, Qingdao, September 15-20, 1996.

[10] L.L.Xie and L.Guo, Fundamental limitations of discrete-time adaptive nonlinear control, IEEE Trans. Automat. Contr., to appear, 1998.

[11] K.S. Narendra and K.Parthasarathy, Identification and control of dynamical systems using neural networks, IEEE Trans. Neural Networks, vol.1, pp.4-27, 1990.

[12] K.Zhou, J.C.Doyle and K.Glover, Robust and Optimal Control, NJ: Prentice Hall, 1996. 